\title{
Dynamic SPECT Measurement of Absolute Myocardial Blood Flow in a Porcine Model
}

\author{
R. Glenn Wells ${ }^{1}$, Rachel Timmins ${ }^{1}$, Ran Klein ${ }^{1}$, Julia Lockwood ${ }^{1}$, Brian Marvin ${ }^{1}$, Robert A. deKemp ${ }^{1}$, Lihui Wei ${ }^{2}$, \\ and Terrence D. Ruddy ${ }^{1}$ \\ ${ }^{1}$ Division of Cardiology, University of Ottawa Heart Institute, Ottawa, Ontario, Canada; and ${ }^{2}$ Nordion Inc., Ottawa, Ontario, Canada
}

\begin{abstract}
Absolute myocardial blood flow (MBF) and myocardial flow reserve (MFR) provide incremental diagnostic and prognostic information over relative perfusion alone. Recent development of dedicated cardiac SPECT cameras with better sensitivity and temporal resolution make dynamic SPECT imaging more practical. In this study, we evaluate the measurement of MBF using a multipinhole dedicated cardiac SPECT camera in a pig model of rest and transient occlusion at stress using 3 common tracers: ${ }^{201} \mathrm{Tl}$, 99mTc-tetrofosmin, and 99mTc-sestamibi. Methods: Animals $(n=$

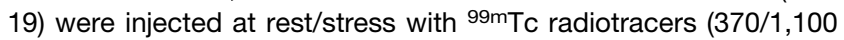
$\mathrm{MBq})$ or ${ }^{201} \mathrm{TI}(37 / 110 \mathrm{MBq})$ with a 1 -h delay between rest and dipyridamole stress. With each tracer, microspheres were injected simultaneously as the gold standard measurement for MBF. Dynamic images were obtained for 11 min starting with each injection. Residual resting activity was subtracted from stress data and images reconstructed with $\mathrm{CT}$-based attenuation correction and energy window-based scatter correction. Dynamic images were processed with kinetic analysis software using a 1-tissue-compartment model to obtain the uptake rate constant $K_{1}$ as a function of microsphere MBF. Results: Measured extraction fractions agree with those obtained previously using ex vivo techniques. Converting $K_{1}$ back to MBF using the measured extraction fractions produced accurate values and good correlations with microsphere MBF: $r=$ $0.75-0.90(P<0.01$ for all). The correlation in the MFR was between $r=0.57$ and $0.94(P<0.01)$. Conclusion: Noninvasive measurement of absolute MBF with stationary dedicated cardiac SPECT is feasible using common perfusion tracers.
\end{abstract}

Key Words: SPECT; absolute myocardial blood flow; myocardial perfusion imaging; pig model

J Nucl Med 2014; 55:1685-1691

DOI: 10.2967/jnumed.114.139782

$\mathbf{S}$ using PET have demonstrated that absolute myocardial blood flow (MBF) and myocardial flow reserve (MFR = stress/ rest $\mathrm{MBF}$ ) provide incremental diagnostic and prognostic information over relative perfusion alone (1-4). Imaging of myocardial perfusion is much more commonly performed with SPECT than with PET, but MBF measurements are not typically acquired.

Received Feb. 27, 2014; revision accepted Jul. 28, 2014.

For correspondence contact: R. Glenn Wells, H2103c, Cardiac PET Research, University of Ottawa Heart Institute, 40 Ruskin St., Ottawa, ON, Canada, K1Y 4W7.

E-mail: gwells@ottawaheart.ca

Published online Sep. 4, 2014.

COPYRIGHT (c) 2014 by the Society of Nuclear Medicine and Molecular Imaging, Inc.
Measuring MBF is difficult with standard SPECT cameras because of the need for attenuation and scatter correction and the need to rotate around the patient for 3-dimensional imaging. Recent studies have shown that it is possible to obtain an index of the MFR without a direct measure of MBF using a combination of dynamic planar followed by static SPECT acquisitions (5) and that this can provide some prognostic information (6). Other studies have demonstrated that rapid camera rotation can provide dynamic tomographic data and hence a measure of MFR (7) and the arterial input function (8), suggesting that accurate measures of MBF could be possible (9). The practicality of measuring MBF has increased greatly, however, with the advent of dedicated cardiac cameras.

Dedicated cardiac cameras such as the DSPECT system (Spectrum Dynamics Medical Inc.) or the Discovery NM 530c/ 570c cameras (GE Healthcare) have greatly improved sensitivity and do not rotate around the patient (10). These features allow dynamic imaging with high temporal resolution that is supported by improved count density and raises the possibility of clinically practical MBF measurement with SPECT. Recent work measured an index of global MFR (11), but no studies have measured MBF. Studies have focused on the use of ${ }^{99 \mathrm{~m} T c-s e s t a m i b i}$ and ${ }^{99 \mathrm{~m}} \mathrm{Tc}-$ tetrofosmin as they are more common and data from these tracers have much higher count statistics than ${ }^{201} \mathrm{Tl}$. However, both ${ }^{99 \mathrm{~m}} \mathrm{Tc}$ tracers have low extraction fractions (EFs) at high flow rates, limiting the apparent increase in MBF between rest and stress. ${ }^{201} \mathrm{Tl}$ has a better EF but the high dosimetry of the tracer leads to increased image noise and reduced image quality. Thus, it is unclear which tracer would provide the most accurate MBF.

In this study, we evaluate the measurement of absolute MBF using a multipinhole cardiac SPECT camera in a pig model of rest followed by transient occlusion at stress using a clinically feasible acquisition protocol and clinically appropriate doses of 3 common tracers: ${ }^{201} \mathrm{Tl}$, ${ }^{99 \mathrm{~m}} \mathrm{Tc}$-tetrofosmin, and ${ }^{99 \mathrm{~m}} \mathrm{Tc}$-sestamibi.

\section{MATERIALS AND METHODS}

A pig model of transient occlusion of the left anterior descending (LAD) coronary artery at stress similar to Nekolla et al. (12) was used to produce a broad range of flow with regional variation. Farm-bred Yorkshire-cross female pigs (30-40 kg) were anesthetized with tiletamine, zolazepam (Fort Dodge), and xylazine (Bayer) and maintained using $2 \%-3 \%$ isoflurane. A thoracotomy was performed and a suture loop placed around the LAD just below the second diagonal branch. Suture lines were brought out of the chest, allowing occlusion and release during imaging. For the normal animals, the same surgery was performed but the suture loop was not placed. The number of occluded and normal pigs that completed the protocol was, respectively, 6 and 4 $\left.{ }^{201} \mathrm{Tl}\right), 4$ and 5 ( ${ }^{99 \mathrm{~m}} \mathrm{Tc}$-tetrofosmin), and 3 and 1 (99m Tc-sestamibi). Of these, we excluded 4 studies because the average microsphere 
results in the nonoccluded heart regions were more than 2.3 SDs (13) away from the mean values normally seen with this pig model, as assessed with a separate cohort of 21 animals. The range of microsphere values accepted for rest MBF, stress MBF, and MFR were 0.2$1.7 \mathrm{~mL} / \mathrm{min} / \mathrm{g}, 1.5-4.1 \mathrm{~mL} / \mathrm{min} / \mathrm{g}$, and $1.1-5.1$, respectively. The excluded studies were 1 occluded ${ }^{99} \mathrm{~m}$ Tc-tetrofosmin study, 1 normal ${ }^{201} \mathrm{Tc}$ study, and 2 occluded ${ }^{201} \mathrm{Tl}$ studies. The study was performed with the approval of the University of Ottawa Animal Care Ethics Committee in accordance with the guidelines of the Canadian Council on Animal Care.

Images were acquired on a Discovery NM 530c (GE Healthcare) camera; additional details are in the Supplemental Methods (available at http://jnm.snmjournals.org) (14). For all tracers, acquisition mimicked a 1 -d rest/stress ${ }^{99 \mathrm{~m}} \mathrm{Tc}$-tetrofosmin human imaging protocol. The animal was positioned supine in the camera, and for the ${ }^{99 \mathrm{~m}} \mathrm{Tc}$ studies, 370 and $1,100 \mathrm{MBq}(10$ and $30 \mathrm{mCi})$ were injected at rest and stress, respectively, whereas the ${ }^{201} \mathrm{Tl}$ studies used 37 and $110 \mathrm{MBq}$ (1 and $3 \mathrm{mCi}$ ), respectively. Tracer was delivered intravenously using a Harvard pump (Harvard Apparatus) as a 9-mL bolus over $20 \mathrm{~s}$, followed by a 9-mL saline flush over $20 \mathrm{~s}$. Gold (rest) or samarium (stress) neutron-activated microspheres (BioPal Inc.) were injected by hand over $10 \mathrm{~s}$ into the left atrium at the same time as the tracer. For each, blood was withdrawn from a femoral artery for $4 \mathrm{~min}$ at $4 \mathrm{~mL} / \mathrm{min}$ starting just before microsphere injection. Image data were acquired in list-mode for 11 min starting just before tracer injection. Resting images were acquired first and repeated just before stress. Stress was induced pharmacologically $1 \mathrm{~h}$ after the rest injection using dipyridamole $(0.14 \mathrm{mg} / \mathrm{kg} / \mathrm{min}$ over $4 \mathrm{~min})$. Phenylephrine was given as needed to maintain blood pressure. At 4 min after dipyridamole, the suture was tightened, occluding the LAD. Thirty seconds after occlusion, tracer and microspheres were injected. Two minutes after tracer injection, the occlusion was released. For each animal, a CT scan $(120 \mathrm{kVp}, 1.0 \mathrm{~mA}, 2.0 \mathrm{rpm})$ was acquired for attenuation correction (AC) on an Infinia-Hawkeye 4 SPECT/CT camera (GE Healthcare), with the pig repositioned in the same orientation as in the NM 530c camera. For the normal (nonoccluded) animals, the same imaging procedure was used except that no occlusion was performed.

Immediately after the CT scan, the pig was euthanized and the heart extracted and sectioned. Similar to DaSilva et al. (15), the heart was sliced perpendicular to the long axis and divided into the apex and 4 additional approximately 1 -cm-thick short-axis slabs. The apex was quartered, and the other slabs were sectioned into approximately 1-g samples after removal of the right ventricle (a total of 44-50 pieces per heart) and numbered clockwise from the LAD junction of the right and left ventricles. The sections were weighed, measured off-site at BioPal Inc., and converted into flow measurements using standard methods (16). Seventeen-segment and 3-vascular-territory polar maps of the microsphere MBF values were generated using the recorded location of each tissue sample.

The acquired list-mode data were rebinned into $9 \times 10,6 \times 15$, and $4 \times 120 \mathrm{~s}$ frames. The initial frame duration was $10 \mathrm{~s}$, similar to our acquisition protocol used for clinical ${ }^{82} \mathrm{Rb}$ PET imaging. The frame duration was increased as the rate of change in the distribution slowed. Projection data for each frame were independently reconstructed using manufacturer-supplied generalized expectationmaximization maximum a posteriori iterative reconstruction (14) without corrections (NC), with CT-based AC, and with $\mathrm{AC}$ and scatter correction (SC) as detailed in the Supplemental Methods. SC was performed on the projection data using a dual-energy-window $\left({ }^{99 \mathrm{~m}} \mathrm{Tc}\right.$ tracers) or triple-energy-window $\left({ }^{201} \mathrm{Tl}\right)$ estimate.

The reconstructed dynamic image series was processed using FlowQuant (University of Ottawa Heart Institute Research Corporation), our semiautomated in-house kinetic analysis software (17).
A 1-tissue-compartment model (CM) was chosen because this model has been shown to be sufficient by others for ${ }^{201} \mathrm{Tl}(18)$ and ${ }^{99 \mathrm{~m}} \mathrm{Tc}-$ sestamibi (11). The $1 \mathrm{CM}$ uptake rate constant, $K_{1}$, is related to MBF by:

$$
K_{1}=\mathrm{EF}(\mathrm{MBF}) \mathrm{MBF}=\left(1-e^{(-[\alpha \mathrm{MBF}+\beta] / \mathrm{MBF})}\right) \mathrm{MBF} . \quad \text { Eq. } 1
$$

Equation 1 assumes a generalized Renkin-Crone model for the EF inwhich $\alpha$ and $\beta$ are parameters to be fit to the data, and $\alpha \mathrm{MBF}+\beta$ corresponds to the permeability-surface-area product of the capillaries.
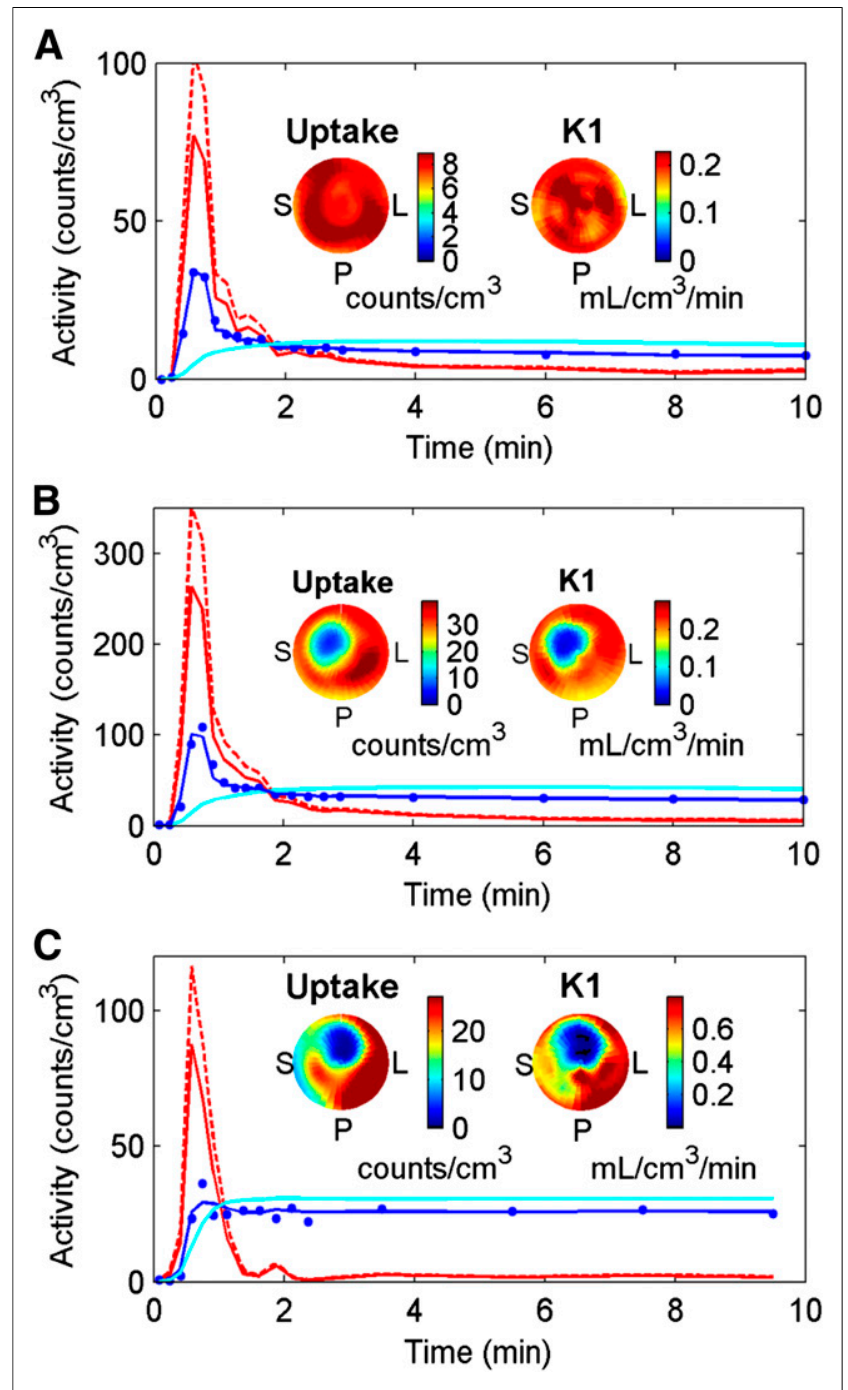

FIGURE 1. Representative time-activity curves from attenuation- and

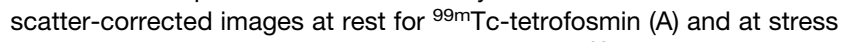
following subtraction of residual rest uptake for ${ }^{99 m T c}$-tetrofosmin (B) and ${ }^{201} \mathrm{TI}(\mathrm{C})$. Shown are arterial whole-blood concentrations (solid red) measured from region in left ventricle, plasma concentration or arterial blood curve corrected for tracer-blood binding (dashed red), CM fit (solid blue) to sampled values (dots) from high-count region of myocardium, and resultant estimated pure myocardium tissue curve (cyan) after correction for spillover and partial-volume effects. Also shown are uptake and $K_{1}$ polar maps. Higher EF of ${ }^{201} \mathrm{TI}$ increases $K_{1}$ value and

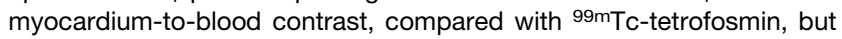
${ }^{201} \mathrm{TI}$ images have increased noise (Table 1), as reflected by area of reduced uptake in right coronary artery territory of ${ }^{201} \mathrm{TI}$ polar map. 
TABLE 1

Homogeneity of Uptake Rate Constant $K_{1}$ from 17-Segment Analysis

\begin{tabular}{|c|c|c|c|c|c|c|}
\hline Tracer & $\begin{array}{l}\text { Mean rest } K_{1} \pm S D \\
(\mathrm{~mL} / \mathrm{min} / \mathrm{g})\end{array}$ & Rest COV (\%) & $\begin{array}{c}\text { Mean stress } \\
K_{1}{ }^{\dagger} \pm \mathrm{SD}(\mathrm{mL} / \mathrm{min} / \mathrm{g})\end{array}$ & $\begin{array}{l}\text { Stress } \\
\text { COV (\%) }\end{array}$ & $\begin{array}{c}\text { Mean } \\
\mathrm{MFR}^{\star \dagger} \pm \mathrm{SD}\end{array}$ & $\begin{array}{c}\mathrm{MFR}^{\star} \\
\operatorname{COV}(\%)\end{array}$ \\
\hline \multicolumn{7}{|l|}{${ }^{201} \mathrm{Tl}$} \\
\hline $\mathrm{NC}$ & $0.51 \pm 0.07$ & 13.2 & $0.99 \pm 0.18$ & 20.5 & $1.91 \pm 0.41$ & 22.2 \\
\hline$A C$ & $0.47 \pm 0.07$ & 14.3 & $0.85 \pm 0.15$ & 18.7 & $1.83 \pm 0.32$ & 17.2 \\
\hline ACSC & $0.41 \pm 0.08$ & 19.1 & $0.77 \pm 0.18$ & 23.0 & $2.03 \pm 0.45$ & 21.5 \\
\hline \multicolumn{7}{|c|}{ 99mTc-tetrofosmin } \\
\hline $\mathrm{NC}$ & $0.25 \pm 0.04$ & 15.1 & $0.33 \pm 0.05$ & 13.9 & $1.38 \pm 0.15$ & 11.5 \\
\hline$A C$ & $0.22 \pm 0.02$ & 8.9 & $0.29 \pm 0.03$ & 9.1 & $1.32 \pm 0.13$ & 10.2 \\
\hline ACSC & $0.21 \pm 0.02$ & 11.8 & $0.28 \pm 0.04$ & 13.5 & $1.33 \pm 0.16$ & 12.3 \\
\hline \multicolumn{7}{|c|}{ 99mTc-sestamibi } \\
\hline $\mathrm{NC}$ & $0.34 \pm 0.04$ & 12.2 & $0.44 \pm 0.08$ & 15.5 & $1.38 \pm 0.26$ & 19.2 \\
\hline$A C$ & $0.28 \pm 0.03$ & 10.3 & $0.40 \pm 0.06$ & 13.3 & $1.42 \pm 0.23$ & 16.0 \\
\hline ACSC & $0.27 \pm 0.03$ & 12.6 & $0.39 \pm 0.06$ & 15.2 & $1.49 \pm 0.28$ & 18.2 \\
\hline
\end{tabular}

The FlowQuant software estimated $K_{1}$ in each of 576 myocardial polar-map sectors using nonlinear weighted least-squares regression with weighting proportional to the frame duration. The goodness-of-fit was evaluated with reduced $\chi^{2}$ and coefficient of determination measures. The $K_{1}$ polar map was then collapsed into either a 17 -segment representation (19) or 3 coronary vascular territories for comparison to the microsphere data. The Spearman rank correlation between $K_{1}$ and microsphere MBF was measured. The SPECT $K_{1}$ and microsphere MBF data were then fit to Equation 1 using a nonlinear leastsquares algorithm to determine the parameters $\alpha$ and $\beta$. The fitted function was used to convert SPECT $K_{1}$ to MBF, and the resultant Pearson correlation to microsphere $\mathrm{MBF}$ was measured. The 17segment model provided a finer sampling of the data and thus a greater range of flow values and a measure of the variability within the normal or nonoccluded regions of each heart. However, using smaller samples increases the noise in the data, and-given the spatial resolution of SPECT-the samples cannot be considered independent, which may bias measures of correlation. Therefore, we also averaged the data over the 3 coronary vascular territories and repeated our analysis.

Comparisons between reconstruction methods were done using Bonferroni-corrected paired $t$ tests. Comparisons between tracers were done with an unpaired $t$ test. A $P$ value of less than 0.05 was considered significant. The noise level in the measured data was calculated as the coefficient of variation (COV) for the 17-segment data: the $\mathrm{SD}$ divided by the mean of the nonoccluded segments (microsphere MFR $\geq 1.5$ ) of the heart.

\section{RESULTS}

There was little change in the pig heart rate or blood pressure between rest and stress (Supplemental Table 1). The maximum counting rate recorded in the studies was $106 \mathrm{kcps}$ at stress and $35 \mathrm{kcps}$ at rest, well below the camera counting rate limit. The total counts recorded in the last 10-s
FIGURE 2. 17-segment comparison of ${ }^{201} \mathrm{TI}$, ${ }^{99 \mathrm{mTC}}$-tetrofosmin, and ${ }^{99 \mathrm{mTC}} \mathrm{Tc}$-sestamibi estimates of $K_{1}$ to microsphere MBF at rest (A), at stress (B), and for MFR*, compared with microsphere MFR (C). Reconstructions were with ACSC. 
TABLE 2

Spearman Rank Correlation Coefficients for Tracer $K_{1}$ Values, Compared with Microspheres

\begin{tabular}{|c|c|c|c|c|c|c|c|}
\hline \multirow[b]{2}{*}{ Tracer } & \multirow[b]{2}{*}{ Measurement } & \multicolumn{3}{|c|}{17 segments } & \multicolumn{3}{|c|}{3 coronary territories } \\
\hline & & NC & $A C$ & ACSC & NC & $A C$ & ACSC \\
\hline \multirow[t]{2}{*}{${ }^{201} \mathrm{TI}$} & Rest + stress $K_{1}$ & 0.84 & 0.76 & 0.82 & 0.86 & 0.81 & 0.86 \\
\hline & $\mathrm{MFR}^{\star}$ & 0.82 & 0.79 & 0.83 & 0.79 & 0.82 & 0.84 \\
\hline \multirow[t]{2}{*}{ 99mTc-tetrofosmin } & Rest + stress $K_{1}$ & 0.70 & 0.70 & 0.67 & 0.68 & 0.70 & 0.65 \\
\hline & $\mathrm{MFR}^{\star}$ & 0.50 & 0.30 & 0.46 & 0.62 & 0.38 & 0.55 \\
\hline \multirow[t]{2}{*}{ 99mTc-sestamibi } & Rest + stress $K_{1}$ & 0.67 & 0.71 & 0.72 & 0.51 & 0.56 & 0.68 \\
\hline & $\mathrm{MFR}^{\star}$ & 0.88 & 0.81 & 0.83 & 0.80 & 0.80 & 0.90 \\
\hline
\end{tabular}

All correlations are significant $(P<0.01)$.

frame averaged 127 and 31 kilocounts at rest for ${ }^{99 \mathrm{~m}} \mathrm{Tc}$ tracers and ${ }^{201} \mathrm{Tl}$, respectively, and 438 and 144 kilocounts at stress for ${ }^{99 \mathrm{~m}} \mathrm{Tc}$ tracers and ${ }^{201} \mathrm{Tl}$, respectively. In all cases, the myocardial curve showed a good fit to the measured values using the $1 \mathrm{CM}$ (Fig. 1), and there was no significant difference in the reduced $\chi^{2}$ $(P>0.24)$ between the reconstruction methods. The reduced $\chi^{2}$ for the fit to the stress data averaged $0.99 \pm 0.06$ overall and $1.03 \pm 0.06,0.95 \pm 0.02$, and $0.99 \pm 0.06$ for ${ }^{201} \mathrm{Tl},{ }^{99 \mathrm{~m}} \mathrm{Tc}-$ tetrofosmin, and ${ }^{99 \mathrm{~m} T c-s e s t a m i b i, ~ r e s p e c t i v e l y . ~ T h e ~ v a l u e s ~ f o r ~}$ rest were similar. The coefficient of determination $\left(R^{2}\right)$ of the $\mathrm{NC}$ fit were similar for the ${ }^{99 \mathrm{~m}} \mathrm{Tc}$ tracers, averaging $0.92 \pm 0.06$ (rest) and $0.94 \pm 0.02$ (stress), and for ${ }^{201} \mathrm{Tl}$ were $0.80 \pm 0.08$ (rest) and $0.93 \pm 0.03$ (stress). $R^{2}$ values close to 1 suggest that the $1 \mathrm{CM}$ was adequate to describe the shape of the measured time-activity curves. The $R^{2}$ values for AC were similar to NC, but the values for AC and scatter correction (ACSC) were lower, reflecting the increase in noise due to $\mathrm{SC}$; the ${ }^{99 \mathrm{~m}} \mathrm{Tc}$ tracers averaged $0.88 \pm 0.04$ (rest, $P=0.02$ vs. NC) and $0.91 \pm$ 0.03 (stress, $P=0.05$ vs. NC) and for ${ }^{201} \mathrm{Tl}$ the $R^{2}$ was 0.44 \pm 0.13 (rest, $P<0.01$ vs. NC) and $0.74 \pm 0.06$ (stress, $P<$ 0.01 vs. NC). Despite lower $R^{2}$, the $K_{1}$ values for ${ }^{201} \mathrm{Tl}$ with ACSC were still estimated with good precision (Table 1), and the COV was not significantly more than that for NC.

The uptake rate constants $K_{1}$ for the 3 tracers correlated well with microsphere MBF, based on ACSC reconstructions and 17segment analysis (Fig. 2). The results were similar for NC and AC reconstructions and for the 3-coronary-territory analysis (Supplemental Figs. 2-7). The average COV values for $K_{1}$-rest, $K_{1}$-stress, and the ratio of stress/rest $K_{1}\left(\mathrm{MFR}^{*}\right)$ were $13 \%-23 \%$ for ${ }^{201} \mathrm{Tl}$ and $9 \%-19 \%$ for the ${ }^{99 \mathrm{~m}} \mathrm{Tc}$ tracers (Table 1). The average COV for the resting microsphere flow measurement was $14 \%$. The Spearman rank correlations $(\rho)$ (Table 2) were good for ${ }^{201} \mathrm{Tl}$, with a $\rho$ of 0.76 or greater for $K_{1}$ versus microsphere MBF and a $\rho$ of 0.79 or greater for MFR* versus MFR. For ${ }^{99 \mathrm{~m}} \mathrm{Tc}$-tetrofosmin, the correlation was also good for $K_{1}(\rho \geq 0.65)$, though MFR* was lower $(0.30 \leq \rho \leq 0.62)$. For ${ }^{99 \mathrm{~m}} \mathrm{Tc}$-sestamibi, the correlation was good for MFR* $(\rho \geq 0.80)$ and $K_{1}$ with the 17-segment analysis $(\rho \geq$ 0.67 ) but lower for $K_{1}$ with the 3-territory analysis $(\rho \geq 0.51)$.

The combined 17-segment rest and stress $K_{1}$ data from the ACSC images were fit to the microsphere flow values using Equation 1 (Fig. 3) and a similar fit was performed with the 3-coronaryterritory data (Supplemental Fig. 8). The resulting fits (Table 3) produced EFs that matched well with the literature (Fig. 4).

After correction of $K_{1}$ to MBF, the slopes of the linear fits of 17segment (Fig. 5) and 3-coronary-territory (Supplemental Fig. 9) SPECT MBF data to microsphere MBF were between 1.01 and 1.04. The Pearson correlation coefficients for the 17-segment MBF were all good: $r=0.75-0.83$ (Table 3). For MFR, the correlation coefficient was $r=0.57$ for ${ }^{99 \mathrm{~m}} \mathrm{Tc}$-tetrofosmin, $r=0.79$ for ${ }^{201} \mathrm{Tl}$, and $r=0.89$ for ${ }^{99 \mathrm{~m}} \mathrm{Tc}$-sestamibi. For the 3-coronary territory analysis, the MBF Pearson correlation coefficients were $r=0.79-90$ and the MFR correlations were $r=0.62-0.94$.
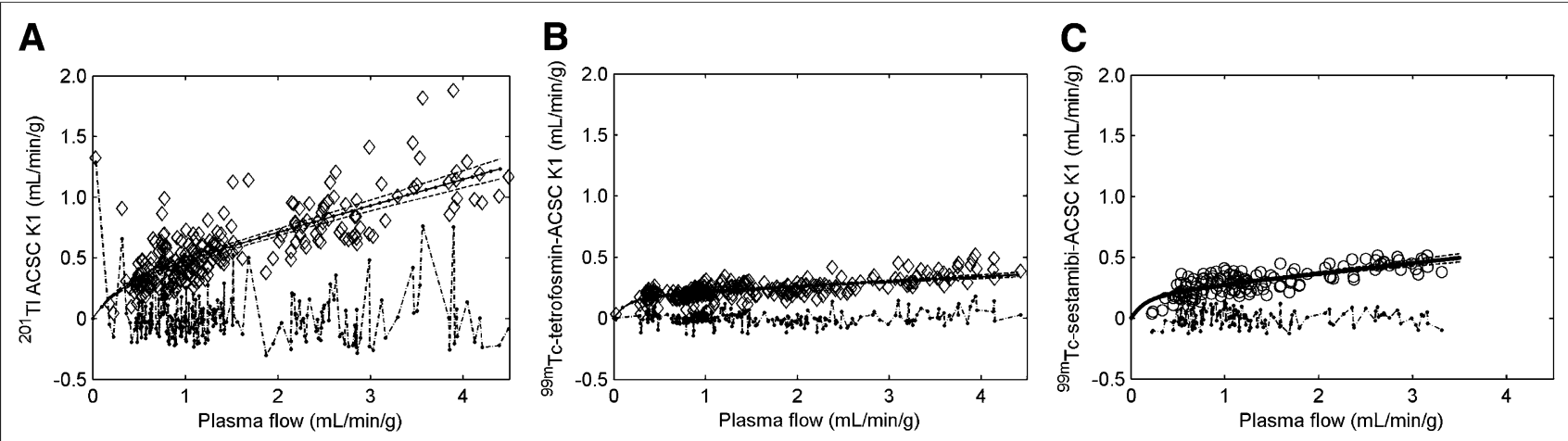

FIGURE 3. 17-segment nonlinear least-squares fits of $K_{1}$ for rest and stress as function of microsphere MBF for ${ }^{201} \mathrm{TI}(\mathrm{A}),{ }^{99 \mathrm{~m} T c-t e t r o f o s m i n}(\mathrm{~B})$, and ${ }^{99 m}$ TC-sestamibi (C). Images were reconstructed with ACSC. Dashed lines show 95\% confidence limit on fit (solid line), and residuals are shown with dash-dot curve. 
TABLE 3

Tracer EF Function and Comparison to Microsphere MBF

\begin{tabular}{|c|c|c|c|c|c|}
\hline Tracer & $a$ & $\beta$ & $R^{2}$ value of fit & Pearson $(r)$ for MBF & Pearson $(r)$ for MFR \\
\hline \multicolumn{6}{|l|}{17 segments } \\
\hline${ }^{201} \mathrm{TI}$ (ACSC) & 0.4117 & 0.4390 & 0.70 & 0.83 & 0.79 \\
\hline${ }^{201} \mathrm{TI}(\mathrm{ACSC})-2 \mathrm{CM}^{*}$ & 0.4830 & 0.8010 & 0.70 & 0.83 & 0.78 \\
\hline 99mTc-tetrofosmin (ACSC) & 0.0660 & 0.2115 & 0.51 & 0.75 & 0.57 \\
\hline 99mTc-sestamibi (ACSC) & 0.1137 & 0.2864 & 0.61 & 0.78 & 0.89 \\
\hline \multicolumn{6}{|l|}{3 coronary territories } \\
\hline 201TI (ACSC) & 0.4222 & 0.3952 & 0.81 & 0.90 & 0.83 \\
\hline${ }^{201} \mathrm{TI}(\mathrm{ACSC})-2 \mathrm{CM}$ & 0.4871 & 0.7471 & 0.81 & 0.90 & 0.83 \\
\hline 99mTc-tetrofosmin (ACSC) & 0.0696 & 02063 & 0.50 & 0.79 & 0.62 \\
\hline 99mTc-sestamibi (ACSC) & 0.1333 & 0.2599 & 0.60 & 0.82 & 0.94 \\
\hline
\end{tabular}

${ }^{*}$ Fitting to $K_{1} / 0.8$ to approximate 2-tissue-compartment fit from 1-tissue-compartment fit (18).

a, $\beta=$ parameters of fit to Equation $3 ; \mathrm{CM}=$ compartment model; $R^{2}=$ coefficient of determination of fit.

All correlations are significant at $P<0.01$.

\section{DISCUSSION}

This study demonstrates, for the first time to our knowledge, the accurate quantification of MBF in an ischemic pig model using attenuation- and scatter-corrected dynamic SPECT imaging with ${ }^{201} \mathrm{Tl}$, ${ }^{99 \mathrm{~m}} \mathrm{Tc}$-sestamibi, and ${ }^{99 \mathrm{~m}} \mathrm{Tc}$-tetrofosmin on a cardiac-dedicated scanner.

$\mathrm{AC}$ and SC improve the accuracy of the images but also increase noise. Image noise varied with the radiotracer and reconstruction method as shown by the COV in Table 1 . Considering all of the tracers combined, at stress $\mathrm{AC}$ has a lower $\mathrm{COV}$ than NC $(P=0.02)$ or ACSC $(P<0.01)$ and ACSC is not significantly different from NC $(P=0.56)$. The results for rest and MFR were similar. Thus, the increase in noise with AC was more than offset by the reduction in attenuation artifacts but, whereas SC may improve accuracy, it came at the cost of increasing COV back to NC levels. Reducing noise by including scatter within the reconstruction or using more advanced multidimensional reconstruction algorithms (9) might thus be beneficial. When ${ }^{201} \mathrm{Tl}$ was compared with ${ }^{99 \mathrm{~m}} \mathrm{Tc}$-tetrofosmin, the noise levels were not significantly different with $\mathrm{NC}(P>0.18)$, but ${ }^{201} \mathrm{Tl}$ had significantly higher COV with AC and ACSC $(P<0.02)$. These differences reflect the increased noise in the ${ }^{201} \mathrm{Tl}$ images caused by a reduced amount of injected tracer. The rank correlations of $K_{1}$ and MFR* with microsphere MBF and MFR (Table 2) were similar for all reconstruction methods.

The ${ }^{99 m}$ Tc-tetrofosmin and ${ }^{99 m}$ Tc-sestamibi results (Figs. 2 and 3) showed the expected reduction in measured $K_{1}$ and MFR* at higher blood flow values (20). Thus, although the noise levels in the ${ }^{99 \mathrm{~m}} \mathrm{Tc}$ datasets were lower than with ${ }^{201} \mathrm{Tl}$, the ratio of normal stress over rest flow (MFR) was also lower, producing a similar signal-to-noise ratio. For NC, AC, and ACSC in the nonoccluded segments of the heart, both ${ }^{201} \mathrm{Tl}$ and ${ }^{99 \mathrm{~m}} \mathrm{Tc}-$ tetrofosmin produced average MFR*s that were 2.1-2.6 SDs above 1, and for ${ }^{99 \mathrm{~m} T c-s e s t a m i b i}$ they were 1.5-1.8 SDs above 1 (Table 1). The average measured MFR* value for ${ }^{99 \mathrm{~m} T c-s e s t a m i b i ~(1.3-}$ 1.5 ) is in agreement with other studies that have measured MFR* with SPECT using simplified alternative methods $(5,11)$. Benefiting from the higher mean MFR* values for ${ }^{201} \mathrm{Tl}$ will require improved techniques to reduce noise such as prior-based multidimensional reconstruction (9).

The injected activities used in these studies were typical of 1-d human clinical protocols. The pigs in this study were smaller than typical patients, resulting in less attenuation and scatter and leading to better-quality images for dynamic processing. In addition, there is increasing motivation to capitalize on the dedicated cardiac camera's increased sensitivity to reduce the patient radiation exposure. These factors may lead to a decreased

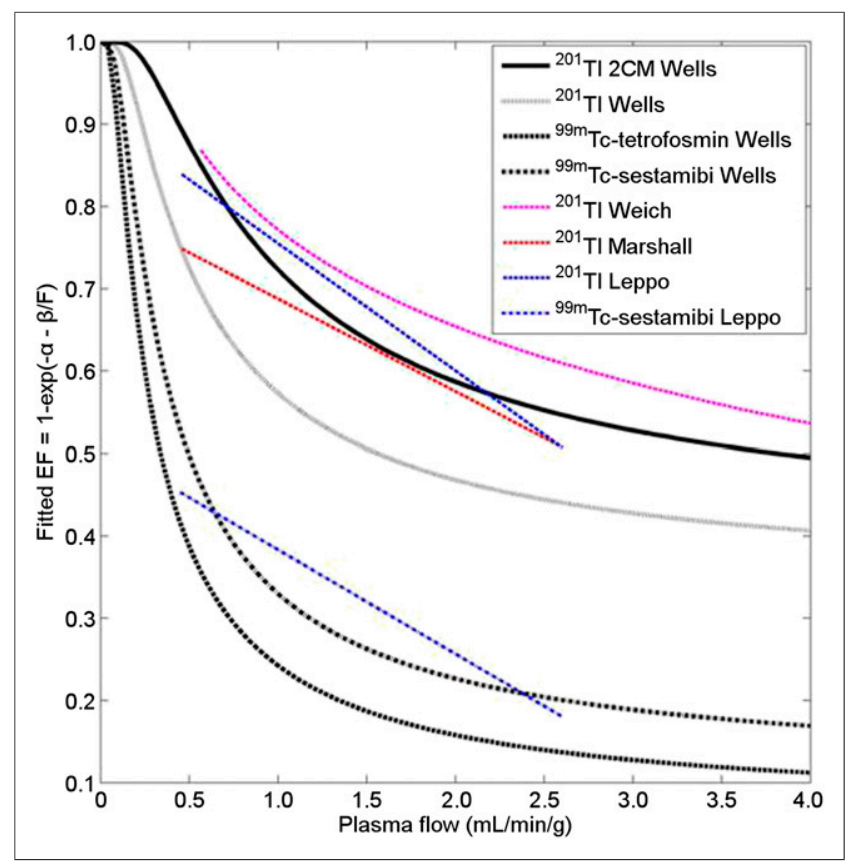

FIGURE 4. Comparison of measured $1 \mathrm{CM}$ EFs for ${ }^{201} \mathrm{TI},{ }^{99 \mathrm{mT}} \mathrm{Tc}$-tetrofos-

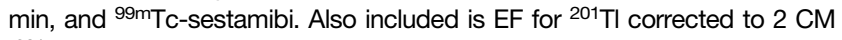
$\left({ }^{201} \mathrm{TI} 2 \mathrm{CM}\right)$ by scaling $K_{1}$ by $1 / 0.8$ (18). These EFs are compared with literature values for ${ }^{201} \mathrm{TI}$ from Weich et al. (24), Leppo and Meerdink (25), and Marshall et al. (24) and for ${ }^{99 m}$ Tc-sestamibi from Leppo and Meerdink (25). 


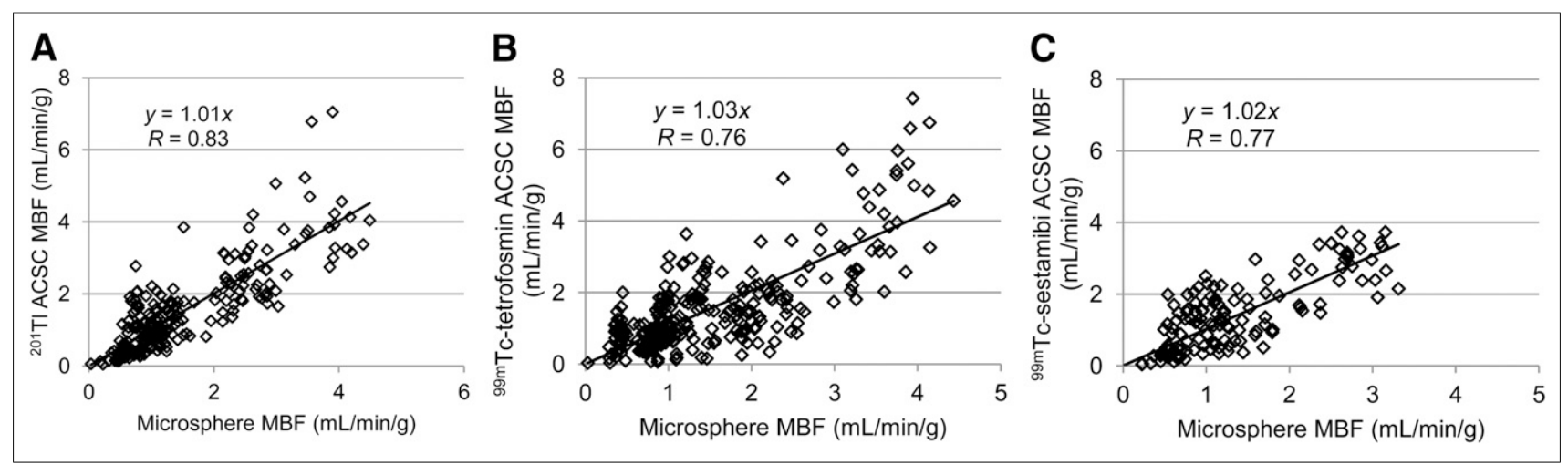

FIGURE 5. 17-segment comparison of microsphere MBF to MBF measured with attenuation- and scatter-corrected ${ }^{201} \mathrm{TI}(\mathrm{A})$, ${ }^{99 \mathrm{~m}} \mathrm{TC}$-tetrofosmin (B), and ${ }^{99 \mathrm{~m} T c}$-sestamibi (C). $K_{1}$ values were converted to MBF using the fitted functions shown in Figure 3.

signal-to-noise when applying this approach to human studies. Evaluation in humans against an accepted gold standard such as quantitative PET perfusion imaging is needed before considering implementation of this technique for routine clinical SPECT flow measurement.

In this study, the tracer was injected as an extended bolus over $20 \mathrm{~s}$. Our typical clinical protocol for ${ }^{82} \mathrm{Rb}$ flow imaging with PET uses a constant-activity-rate injection over $30 \mathrm{~s}$ whereas others have suggested a much more rapid injection (11). Previous work with PET has shown that use of longer tracer injection times can improve the quantitative accuracy and precision of flow measurements (21). Our data were reprocessed by subdividing the three 10-s frames near the peak of the arterial input function into ten 3-s frames in an effort to better sample the peak. The results (not shown) did not show any improvement in correlation with microsphere flow. However, further work would be needed to determine whether extending the bolus duration could improve the SPECT flow measurements.

Release of the occlusion 2 min after tracer injection will result in a change in flow and potentially reactive hyperemia within the occluded tissues. The uptake of ${ }^{99 \mathrm{~m}} \mathrm{Tc}$ tracers is rapid and static, but ${ }^{201} \mathrm{Tl}$ is known to redistribute after reperfusion (22). Residual tracer in the blood or redistributing tracer during the last 9 min of imaging would be taken up under different flow conditions after reperfusion and this may have degraded the accuracy of ${ }^{201} \mathrm{Tl} \mathrm{MBF}$ measurements, compared with microspheres in the ischemic region.

This work used a $1 \mathrm{CM}$ to fit all of the data for both ${ }^{201} \mathrm{Tl}$ and ${ }^{99 \mathrm{~m}} \mathrm{Tc}$ tracers. Previous work with ${ }^{201} \mathrm{Tl}$ has suggested that although a $2 \mathrm{CM}$ is more accurate physiologically (18), the $1 \mathrm{CM}$ produces $K_{1}$ estimates that are almost as accurate and are related to the $2 \mathrm{CM}$ fits by a simple scaling factor of 0.8 . These results suggest that a $1 \mathrm{CM}$ may be more suitable for practical application for which the noise levels in the images are higher and imaging times are shorter (23). Scaling our $1 \mathrm{CM} K_{1}$ values by $1 / 0.8$ generates an $\mathrm{EF}$ for ${ }^{201} \mathrm{Tl}$ that agrees with the literature (Fig. 4) (2426). However, the number of animals used in our study was limited, and the value of a $1 \mathrm{CM}$ over a $2 \mathrm{CM}$ must still be assessed in more complex disease states such as mixed-normal, ischemic, and infarcted tissues. Considered separately, the ischemic and remote territories at stress were mostly not significantly different from microspheres but showed a trend toward underestimation of MBF that will require further study (Supplemental Table 3). The appropriate model for the ${ }^{99 \mathrm{~m}} \mathrm{Tc}$ tracers has not been extensively explored. However, other studies using a $1 \mathrm{CM}$ with a $k_{2}$ of $0(5,11)$ have demonstrated a successful measurement of global MFR*. In addition, the reduced $\chi^{2}$ values and $R^{2}$ values of the fits to the ${ }^{99 \mathrm{~m}} \mathrm{Tc}$ data suggest that the $1 \mathrm{CM}$ provided a good description of the tracer kinetics in our experiments. Also, the EF for ${ }^{99 m} \mathrm{Tc}-$ sestamibi using the $1 \mathrm{CM}$ agrees with previous literature (Fig. 4) (25). Nevertheless, a more complex model could lead to an improvement in the correlation with microspheres and could be explored in future studies.

This study used a clinically feasible acquisition protocol and clinically appropriate doses of 3 common tracers. However, ${ }^{99 \mathrm{~m}} \mathrm{Tc}$ tracers have limited extraction at high flow rates, and although studies measuring MFR with these tracers are promising $(6,11)$ contrast between stress and resting flows will be less than that typically seen with PET. SPECT also has lower sensitivity than PET, increasing the noise in the images despite the increased sensitivity of the dedicated cardiac SPECT cameras, and SPECT spatial resolution is worse than PET, which increases partial-volume effects. Finally, there were only 19 animals used in the study, divided over 3 tracers. The limited number of samples at the extreme ends of the MBF range may affect the fit and introduce some error into the final measure values. Also, tracer uptake and response to dipyridamole varies among animals and so caution should be exercised to not overgeneralize the results of these experiments. Thus, whereas these pig studies show a promising correlation with microsphere MBF, full validation in humans is needed before we can confidently move to clinical implementation.

\section{CONCLUSION}

We have demonstrated that it is possible, in a pig model of rest and transient occlusion at stress, to use a stationary dedicated cardiac camera to perform dynamic SPECT for the measurement of absolute myocardial blood flow with ${ }^{201} \mathrm{Tl},{ }^{99 \mathrm{~m}} \mathrm{Tc}$-tetrofosmin, and ${ }^{99 \mathrm{~m}} \mathrm{Tc}$-sestamibi. The correlations of the SPECT- to microspherederived flow values were good ( $r \geq 0.75)$. The approach used is readily translated to human studies and could provide incremental diagnostic and prognostic information over relative perfusion alone but will require further validation before it can be clinically implemented.

\section{DISCLOSURE}

The costs of publication of this article were defrayed in part by the payment of page charges. Therefore, and solely to indicate this fact, this article is hereby marked "advertisement" in accordance 
with 18 USC section 1734. This research was supported by grants from the Heart and Stroke Foundation of Canada (NA6939, BR7489) and a grant from the Canadian Institutes of Health Research and the Natural Sciences and Engineering Research Council of Canada (NSERC) (RMIPJ 389641-09). R. Glenn Wells and Terrence D. Ruddy collaborate with GE Healthcare on research projects and have received honoraria for speaking at GE Healthcare User Meetings. Robert deKemp and Ran Klein receive revenues from the sale of FlowQuant. No other potential conflict of interest relevant to this article was reported.

\section{REFERENCES}

1. Herzog BA, Husmann L, Gaemperli O, et al. Long-term prognostic value of ${ }^{13} \mathrm{~N}$ ammonia myocardial perfusion positron emission tomography added value of coronary flow reserve. J Am Coll Cardiol. 2009;54:150-156.

2. Saraste A, Kajander S, Han C, Nesterov SV, Knuuti J. PET: is myocardial flow quantification a clinical reality? J Nucl Cardiol. 2012;19:1044-1059.

3. Murthy VL, Naya M, Foster CR, et al. Association between coronary vascular dysfunction and cardiac mortality in patients with and without diabetes mellitus. Circulation. 2012;126:1858-1868.

4. Valenta I, Dilsizian V, Quercioli A, Ruddy TD, Schindler TH. Quantitative PET/CT measures of myocardial flow reserve and atherosclerosis for cardiac risk assessment and predicting adverse patient outcomes. Curr Cardiol Rep. 2013;15:344-353.

5. Storto G, Sorrentino AR, Pellegrino T, Liuzzi R, Petretta M, Cuocolo A. Assessment of coronary flow reserve by sestamibi imaging in patients with typical chest pain and normal coronary arteries. Eur J Nucl Med Mol Imaging. 2007;34:1156-1161.

6. Daniele S, Nappi C, Acampa W, et al. Incremental prognostic value of coronary flow reserve assessed with single-photon emission computed tomography. J Nucl Cardiol. 2011;18:612-619.

7. Gullberg GT, DiBella EVR, Sinusas AJ. Estimation of coronary flow reserve: can SPECT compete with other modalities? J Nucl Cardiol. 2001;8:620-625.

8. Winant $\mathrm{CD}$, Aparici CM, Zelnik YR, et al. Investigation of dynamic SPECT measurements of the arterial input function in human subjects using simulation, phantom and human studies. Phys Med Biol. 2012;57:375-393.

9. Gullberg GT, Reutter BW, Sitek A, Maltz JS, Budinger TF. Dynamic single photon emission computed tomography: basic principles and cardiac applications. Phys Med Biol. 2010;55:R111-R191.

10. Slomka PJ, Patton JA, Berman DS, Germano G. Advances in technical aspects of myocardial perfusion SPECT imaging. J Nucl Cardiol. 2009;16:255-276.
11. Ben-Haim S, Murthy VL, Breault C, et al. Quantification of myocardial perfusion reserve using dynamic SPECT imaging in humans: a feasibility study. J Nucl Med. 2013;54:873-879.

12. Nekolla SG, Reder S, Saraste A, et al. Evaluation of the novel myocardial perfusion positron-emission tomography tracer ${ }^{18} \mathrm{~F}-\mathrm{BMS}-747158-02$ : comparison to ${ }^{13} \mathrm{~N}$-ammonia and validation with microspheres in a pig model. Circulation. 2009; 119:2333-2342.

13. Taylor JR. An introduction to error analysis. In: The Study of Uncertainties in Physical Measurements. 2nd ed. Sausalito, CA: University Science Books; 1997:166-170.

14. Bocher M, Blevis IM, Tsukerman L, Shrem Y, Kovalski G, Volokh L. A fast cardiac gamma camera with dynamic SPECT capabilities: design, system validation and future potential. Eur J Nucl Med Mol Imaging. 2010;37:1887-1902.

15. Da Silva AJ, Tang HR, Wong KH, Wu MC, Dae MW, Hasegawa BH. Absolute quantification of regional myocardial uptake of ${ }^{99 \mathrm{~m}} \mathrm{Tc}$-sestamibi with SPECT: experimental validation in a porcine model. J Nucl Med. 2001;42:772-779.

16. Heymann MA, Payne BD, Hoffman JIE, Rudolph AM. Blood flow measurements with radionuclide-labeled particles. Prog Cardiovasc Dis. 1977;20:55-79.

17. Klein R, Beanlands RSB, deKemp RA. Quantification of myocardial blood flow and flow reserve: technical aspects. $J$ Nucl Cardiol. 2010;17:555-570.

18. Iida H, Eberl S, Kim K-M, et al. Absolute quantitation of myocardial blood flow with ${ }^{201} \mathrm{Tl}$ and dynamic SPECT in canine: optimization and validation of kinetic modeling. Eur J Nucl Med Mol Imaging. 2008;35:896-905.

19. Cerqueira MD, Weissman NJ, Dilsizian V, et al. Standardized myocardial segmentation and nomenclature for tomographic imaging of the heart: a statement for healthcare professionals from the Cardiac Imaging Committee of the Council on Clinical Cardiology of the American Heart Association. Circulation. 2002;105:539-542.

20. Meleca MJ, McGoron AJ, Gerson MC, et al. Flow versus uptake comparisons of thallium-201 with technetium-99m perfusion tracers in a canine model of myocardial ischemia. J Nucl Med. 1997;38:1847-1856.

21. Klein R, Renaud JM, Ziadi MC, et al. Intra- and inter-operator repeatability of myocardial blood flow and myocardial flow reserve measurements using rubdium- 82 PET and a highly automated analysis program. J Nucl Cardiol. 2010;17:600-616.

22. Beller GA, Watson DD, Ackell P, Pohost GM. Time course of thallium-201 redistribution after transient myocardial ischemia. Circulation. 1980;61:791-797.

23. Coxson PG, Huesman RH, Borland L. Consequences of using a simplified kinetic model for dynamic PET data. J Nucl Med. 1997;38:660-667.

24. Weich HF, Strauss HW, Pitt B. The extraction of thallium-201 by the myocardium. Circulation. 1977;56:188-191.

25. Leppo JA, Meerdink DJ. Comparison of the myocardial uptake of a technetiumlabeled isonitrile analogue and thallium. Circ Res. 1989;65:632-639.

26. Marshall RC, Leidholdt EM Jr, Zhang DY, Barnett CA. The effect of flow on technetium-99m-teboroxime (SQ30217) and thallium-201 extraction and retention in rabbit heart. J Nucl Med. 1991;32:1979-1988. 